\title{
A role for eyebrows in regulating the visibility of eye gaze direction
}

Roger Watt, Ben Craven and Sandra Quinn

Department of Psychology

University of Stirling

Stirling FK9 4LA

UK

Correspondence and requests for materials should be addressed to R.W.

(r.j.watt@stir.ac.uk). 


\section{ABSTRACT}

The human eye is unique amongst those of primates in having white sclera against which the dark iris is clearly visible. This high-contrast structure makes the gaze direction of a human potentially easily perceptible to others. For a social creature such as a human, the ability to perceive the direction of another's gaze may be very useful, since gaze usually signals attention. We report data showing that the accuracy of gaze deviation detection is independent of viewing distance up to a certain critical distance, beyond which it collapses. This is, of itself, surprising since most visual tasks are performed better at closer viewing distances. Our data also show that the critical distance, but not accuracy, is affected by the position of the eyebrows so that lowering the eyebrows reduces the critical distance. These findings show that mechanisms exist by which humans could expand or restrict the availability of their gaze direction to others. A way to regulate the availability of the gaze-direction signal could be an advantage. We show that an interpretation of eyebrow function in these terms provides a novel explanation for several well-known eyebrow actions, including the eyebrow flash. 


\section{INTRODUCTION}

In a highly visual species, such as humans, the direction in which the eyes are pointed serves as a generally honest signal to the direction of attention. Eye gaze direction, particularly whether the eyes are averted or not can be a useful cue for judging a person's emotion (eg, Adams and Kleck, 2003, 2005), as a factor in perceiving facial expression (Ganel et al, 2005) and more generally as an aid in perceiving the person (eg Macrae et al, 2002; Jones et al 2006).

It has been known for a long time that we are able to judge accurately the direction of someone else's gaze, especially if we are the subject of that gaze (Gibson \& Pick, 1963; Cline, 1967). We can be subject to errors when the view of the face is not frontal (eg. Anstis, Mayhew, \& Morley, 1969; Jenkins and Langton, 2003; Langton et al, 2004); and our ability to perceive the direction is poorer when the eye is upside down (Senju and Hasegawa 2006).

Humans are the only primates in which the dark iris is seen against a white sclera through the palpebral fissure (the aperture between the eyelids) (Kobayashi \& Kohshima, 1997); this high-contrast pattern provides a number of potential cues to gaze direction. Kobayashi \& Kohshima suggested that the combination of a dark iris and light sclera may have evolved to provide a cue for gaze direction (an advantage in a highly social and cooperative species such as humans), and also that the darker sclera of other primates may have evolved for the opposite purpose, to make gaze direction obscure, possibly so that predators cannot tell that they have been spotted. Ando (2002) has shown that changing the brightness of the scleral areas can have profound effects on perceived eye gaze direction. 
Viewing distance is known to affect the precision of gaze-direction judgements at least from 0.5m to 4m. (Martin and Jones, 1982). Martin and Jones used a "gazer" as stimulus: a person who was instructed to look at the observer's eyes, left shoulder, chest and hair. The observer was required to say which target was being looked at. The results are reported in a non-parametric manner, and so it is not possible to say how precision is affected by viewing distance.

If gaze-direction judgements are based on the pattern presented by the iris and the sclera, then there will be a viewing distance beyond which this pattern cannot be resolved by the viewer and the gaze-direction signal will no longer be available. There would thus be a limited viewing distance within which the signal is available, perhaps with varying quality.

If advertising one's gaze direction is an advantage in some situations and a disadvantage in others, then it would be valuable to be able to regulate the availability of the gaze signal. One way in which this could be achieved is that the quality of the signal could be controlled by regulating the duration of fixations. Another possibility is that the visibility of the eye could be regulated by means of the eyebrows.

Downward motion of the eyebrow is always accompanied by a corresponding downward movement of the upper eyelid, resulting in a smaller palpebral fissure and making less of the eye visible. It is also possible that the lowered eyebrows - as a nearby high-contrast feature - could interfere with the visibility of the eye regions of the face via visual inhibitory mechanisms (Morrone, Burr, and Maffei, 1982) as well as by partial occlusion of the eye. 
In order to investigate the quality and availability of gaze-direction signals, we did psychophysical experiments to measure gaze direction sensitivity as a function of viewing distance, stimulus duration, and eyebrow position.

\section{EXPERIMENT 1}

In Experiment 1 we measured the effect of viewing distance on the minimum detectable rotation of the eyeball for two eyebrow conditions: eyebrows normal and eyebrows lowered.

\section{(a) Observers}

There were two observers, one of whom was the first author. Both had normal or corrected-to-normal vision. The research reported was approved by the departmental ethics committee.

\section{(b) Stimuli}

The stimuli were a set of full-face photographs of 4 people taken as they looked at a series of targets placed either side of the camera lens. Nearly all faces have asymmetries (hair partings, for example) that may bias subjects' judgements of gaze direction. To help to reduce the effect of these asymmetries, the images were used in both their original form, and also reflected about a vertical axis. In this way, a stimulus of a person looking to the left could be obtained from the original image of the person looking to the left, or from the reflected image of them looking to the right. Thus if the judgements of any of the observers in the actual experiment were influenced by irrelevant facial asymmetries, the effect was at least symmetrical. Examples of these stimuli are shown in Figure 1. The stimuli were presented on a 
Sony TFT LCD monitor. The screen size was $33.5 \mathrm{~cm}$ by $27 \mathrm{~cm}$, and the resolution was 1280 by 1024 pixels. We used two stimulus durations $-1.0 \mathrm{~s}$ and a short duration of nominally 20 msec (2 refresh cycles at $100 \mathrm{~Hz}$ ). The effective viewing distance varied from condition to condition over the range $1 \mathrm{~m}$ to $32 \mathrm{~m}$. Two image sizes were used: one-quarter of life size and one-eighth of life size. Viewing distance was set to distances between $0.25 \mathrm{~m}$ and $4 \mathrm{~m}$ in combination with the two image sizes, to create retinal image sizes that corresponded to the desired effective viewing distances.

[Figure 1 about here]

\section{(c) Procedure}

On each trial the observer was shown an image from the stimulus set and was required to indicate, by pressing a button, whether the person in the image was looking to the left or to the right of straight ahead. The size of the cue on each trial was the angular deviation (from straight ahead) of the eye of the person in the image. At the end of the sequence of trials, a cumulative Gaussian was fitted to the psychometric function. From this curve the magnitude of eye turn which was judged correctly on $84 \%$ of trials was estimated, corresponding to the standard deviation of the observer's internal error distribution. We will call this threshold value the minimum detectable eye turn. The trials were organised into blocks of 88 trials. Within each block of trials, the viewing distance and stimulus duration were fixed, and the stimulus level (degree of eye turn) was varied in 11 steps. Eight responses were obtained for each stimulus level in each block. Blocks of the different combinations of viewing distance and stimulus duration were presented in a random order. Each block was repeated 3 times, making a total of $11 \times 8 \times 3=264$ responses per data point. 
[Figure 2 about here]

\section{(d) Results}

The graphs in Figure 2 show minimum detectable eye turn plotted as a function of viewing distance. Each graph shows data for one observer at one stimulus duration. The two lines on each graph are for the two eyebrow conditions: normal and lowered. For both observers and in all conditions the data show one striking feature: sensitivity to gaze deviation is unaffected by viewing distance, up to a certain critical distance. Beyond this distance, performance gets rapidly worse. These curves could be characterized by two parameters: the optimal sensitivity and the critical distance. Both of these parameters are affected by stimulus duration: reducing stimulus duration from $1000 \mathrm{~ms}$ to $20 \mathrm{~ms}$ increases the minimum detectable eye turn from about $0.8^{\circ}$ to about $1.3^{\circ}$, and causes a decrease in the critical distance from about $15 \mathrm{~m}$ to about $8 \mathrm{~m}$. Lowering the eyebrows does not affect the optimal sensitivity, but it does appear to change the critical viewing distance for short duration stimuli.

\section{(e) Discussion of Experiment 1}

The results of the first experiment suggest strongly that there is a region of space, extending outwards from a person's eyes, within which their eye gaze direction can be accurately judged and beyond which there is almost no information. The finding that the size of this region is reduced by brief exposures suggests that when a person makes a brief fixation, then an observer needs to be quite close to know where they were looking. The results also suggest that the position of the eyebrows can be used to control the critical distance. 


\section{EXPERIMENT 2}

In Experiment 2 we further explored the effect of eyebrow position and stimulus duration on the critical distance. Instead of establishing the critical distance by measuring thresholds at every viewing distance, we chose a level of the cue (eye turn) that would be comfortably above threshold for viewing distances below the critical distance, but definitely below threshold for viewing distances above the critical distance, and measured performance with this fixed cue, as a function of effective viewing distance. As the critical viewing distance is passed, performance will decline abruptly from near-perfect to near-chance. In this way we were able to measure the critical distance without the labour of establishing thresholds at every viewing distance. The chosen size of eye turn was $4^{\circ}$. There were three eyebrow conditions: raised eyebrows, neutral eyebrows, and lowered eyebrows, and 4 stimulus durations.

\section{(a) Observers}

There were two observers, one of whom was the second author. Both had normal or corrected-to-normal vision. The research reported was approved by the departmental ethics committee.

\section{(b) Stimuli}

The stimuli were made by taking photographs of 4 subjects as they looked at marks $4^{\circ}$ either side of a camera lens. The subject sat $80 \mathrm{~cm}$ from the camera, which was at the subject's eye level. A head support and a system of sighting marks was provided so that the subject could accurately align their head to be facing straight at the camera. Each subject had photographs taken with their eyebrows in a neutral position and with eyebrows raised and lowered. The photographs were scaled and cropped to produce images of 341 by 512 pixels. Both original and reflected versions of each image were 
used, as in Experiment 1. Examples of these stimuli are shown in Figure 3. The stimuli were presented on a Sony TFT LCD monitor. The screen size was $33.5 \mathrm{~cm}$ by $27 \mathrm{~cm}$, and the resolution was 1280 by 1024 pixels. The actual viewing distance during the experiment was 1 metre. The images were digitally adjusted in size so that they corresponded to effective viewing distances of $5.0 \mathrm{~m}$ to $23.8 \mathrm{~m}$ in 7 equal multiplicative steps. The image sizes as presented ranged from $256 \times 171$ pixels to $54 \times 36$ pixels. Stimulus durations were $0.1,0.2,0.5$, and $1.0 \mathrm{~s}$.

[Figure 3 about here]

\section{(c) Procedure}

As in Experiment 1, on each trial the observer was shown an image of a face and required to indicate, by pressing a button, whether the person in the image was looking to the left or to the right of straight ahead. The different combinations of gaze direction, eyebrow position, effective viewing distance and stimulus duration were each represented by 10 trials, and all trials were interleaved in one block of 1680 trials. Observers were free to interrupt and resume the experiment whenever they wished. Each observer undertook three blocks of trials. The person appearing in the stimulus image on each trial, and the normal or reflected form of the image, were both chosen randomly, with replacement, on each trial.

\section{(d) Results}

The data were collapsed over gaze direction (trials with leftward gazes being combined with trials with rightward gazes), giving 20 trials for each combination of eyebrow position, viewing distance, and stimulus duration. The percentage of correct responses was calculated for each condition. This process produced a number of 
graphs, each showing percent correct responses as a function of effective viewing distance. There was a graph for each combination of eyebrow position and stimulus duration. Each of these graphs was fitted by a cumulative Gaussian, and the effective viewing distance corresponding to $84 \%$ correct responses was extracted from the fit.

[Figure 4 about here]

The graphs in Figure 4 show the critical viewing distance corresponding to 84\% correct responses, as a function of stimulus duration. Each graph shows data for one observer, for the three eyebrow positions. The error bars are 95\% confidence limits. The open symbols in the graph for BC are cases where performance approached but never went below 84\% - these points therefore represent slight extrapolations from the data. The data show that the critical distance increases as stimulus duration increases, and increases as the eyebrows rise through the low, neutral and raised positions. The actual values of the critical distance are in good agreement with the data from Experiment 1.

\section{DISCUSSION}

The data show that sensitivity to deviation of gaze direction is unaffected by viewing distance up to a certain critical distance, and gets rapidly worse thereafter. The critical distance becomes smaller if the stimulus duration is restricted, and correlates positively with eyebrow height. Short stimulus durations also result in reduced sensitivity within the critical distance, but eyebrow height does not affect sensitivity within the critical distance. 


\section{(a) Detecting gaze direction}

We now consider the kinds of gaze-direction cue that the human eye presents, and discuss how the pattern of our results corresponds to what might be expected if gazedirection judgements were based on these cues. In making these comparisons, we are not aiming to identify a single cue to gaze direction. There may be no such single cue, and of course the freedom of the whole head to rotate complicates the issue considerably.

A simple detection of the displacement of the iris from a reference position would result in eye turn thresholds that were constant when considered in terms of the angle subtended at the viewer's eye by the displacement, but not constant when considered in terms of the angle of eyeball rotation. For example, a typical hyperacuity threshold of 5 arc sec would result in an eye turn threshold of $0.23^{\circ}$ at a conversational distance of $2 \mathrm{~m}$, increasing by about $0.12^{\circ}$ for every extra metre viewing distance. This steady increase of eye turn threshold with viewing distance is clearly inconsistent with our data.

The fixed structures of the eye provide reference markers relative to which the position of the iris can be judged. Considered in this way, judgements of iris position (and hence gaze direction) are akin to bisection acuity tasks, in which the cue is the departure of a marker (a line or a dot) from a central position between two fixed flanking markers. An interesting feature of bisection tasks is that the threshold scales linearly with overall stimulus size, over at least two orders of magnitude (Burbeck, 1987). That is, the threshold deviation from centrality is a constant fraction of the distance between the reference markers. For separations less than about 5 arc min (Burbeck, 1987), performance is more closely a constant distance offset. If 
judgements of iris position behave like bisection thresholds, then the thresholds, measured in terms of eyeball rotation, should not vary with viewing distance, up to a viewing distance of very roughly $10 \mathrm{~m}$, beyond which they should get rapidly worse. This is the general pattern of performance that we observe.

Cues to gaze direction do not need to be spatial. The two visible areas of sclera, one either side of the iris, vary in size as the eyeball rotates. A simple comparison of the total light reflected from the two areas of sclera would therefore provide information about eyeball rotation. Up to the point where the areas of sclera fail to be resolved from each other or from surrounding structures, we would not expect eye turn thresholds based on this cue to vary with distance.

As a way of exploring the effect, we took the images used in Experiment 2 and made a simple simulation of the effects of viewing distance on the visual information available. We scaled the size of the image appropriately for various viewing distances and then filtered the resultant set of images with a filter which models the smallest vertically-oriented receptive fields found in visual cortex V1. We measured the strength of the response of such a filter to each of the two parts of the sclera of the right eye, looking straight ahead. If this smallest filter cannot resolve the sclera, then the information is not available.

[Figure 5 about here]

Figure 5 shows how viewing distance affects the response level of the filter to the two parts of the sclera. The three graphs are for three different positions of the eyebrows. For the temporal sclera (lower response on each graph), the response declines smoothly with viewing distance until it vanishes because the finest-scale filter can no 
longer resolve that portion of the sclera from its surround. Over the same distance range, the response to the nasal sclera is higher and grows slightly before it loses its identity and merges with the response to nearby regions of the nose. The vanishing distances measured in this way are similar to the critical distances measured in Experiment 2. In addition, the figure shows that the vanishing distance depends on eyebrow position, so that the higher the eyebrows, the greater the distance over which the two parts of the sclera can be seen. These characteristics of the simulation correspond well with our experimental data.

Our data have shown that there is a zone within which a good gaze-direction signal is available, and outside which the signal rapidly declines. This discussion has shown that there are potential cues to gaze direction that would result in such a well-defined zone.

\section{(b) Social functions of eyebrows}

The finding that the critical distance, but not sensitivity, is modulated by eyebrow position demonstrates that the eyebrows could be used to control the reception of the gaze-direction signal. There are patterns of normal eyebrow use that make sense in these terms. For example, there is the eyebrow flash, a gesture of greeting, usually from some distance (Eibl-Eibesfeldt, 1972). In this gesture the eyebrows are raised for a fraction of a second and then dropped. This signal is generally held to be a token message of recognition, its use being a convention in the same way that the use of the spoken sound "hello" is a convention. However, if the effect of raising the eyebrows is to extend the distance from which gaze direction can be accurately seen, then the 
eyebrow flash may be more than a convention: it may make the eye gaze direction of the sender briefly visible to the receiver. Since that eye gaze direction is at the receiver, it results in the receiver being given a brief "I am looking at you” signal, which would communicate recognition.

\section{SUMMARY}

Our results are simply summarized by using the concept of a spatial signalling zone within which a person's gaze-direction signal is available to all who choose to receive it. We have shown that such a signalling zone has quite sharp boundaries, and that accuracy for perceiving gaze direction within the zone is constant. The size of the zone can be controlled by actions of the sender, including the use of brief fixations and eyebrow position. The use of brief fixations, which corresponds to brief exposure durations in our experiments, results in a slightly decreased sensitivity to gaze direction as well as a reduced signalling zone. In contrast, lowering the eyebrows results in a smaller signalling zone but without any loss of precision within the zone. 


\section{REFERENCES}

Adams RB Jr, Kleck RE (2003) Perceived gaze direction and the processing of facial displays of emotion. Psychological Science 14 (6), 644-647.

Adams RB Jr, Kleck RE. (2005) Effects of direct and averted gaze on the perception of facially communicated emotion. Emotion, 5, 3-11.

Ando S, (2002) Luminance-induced shift in the apparent direction of gaze. Perception $31,657-674$

Anstis, S.M., Mayhew, J.W., \& Morley, T. (1969) The perception of where a face in a television 'portrait' is looking. Am. J. Psychol. 82, 474-489.

Burbeck, C.A. (1987) Position and spatial frequency in large-scale localisation judgements. Vision Research 27, 417-428.

Cline, M.G. (1967) The perception of where a person is looking. Am. J. Psychol. 80, $41-50$.

Eibl-Eibesfeldt, I. (1972) Similarities and differences between cultures in expressive movements. In Hinde R. (ed.) Non-Verbal Communication. London: Cambridge University Press.

Ekman, P., \& Friesen, W. V. (1977) Nonverbal behavior. In Ostwald, P. (ed.) Communication and Human Interaction New York: Grune \& Stratton. 
Ekman, P. (1979) About brows: Emotional and conversational signals. In von Cranach, M., Foppa, K., Lepenies, W., \& Ploog, D. (eds.) Human Ethology Cambridge: Cambridge University Press.

Ganel T, Goshen-Gottstein Y, Goodale MA. (2005) Interactions between the processing of gaze direction and facial expression. Vision Res. 45,1191-200.

Gibson, J. J. \& Pick, A.D. (1963) Perception of another person’s looking behaviour. Am. J. Psychol. 76, 386-394.

Jenkins J, Langton SR. (2003) Configural processing in the perception of eye-gaze direction. Perception. 32,1181-8.

Jones BC, DeBruine LM, Little AC, Conway AC, and Feinberg DR. (2006) Integrating Gaze Direction and Expression in Preferences for Attractive Faces. Psychological Science 17:7, 588-591

Kendon, A. (1967) Some functions of gaze direction in social interaction. Acta Psychologica 32, 1-25.

Kobayashi, H. \& Kohshima, S. (1997) Unique morphology of the human eye. Nature 387, 767-768.

Langton SR, Honeyman H, Tessler E. (2004) The influence of head contour and nose angle on the perception of eye-gaze direction. Percept Psychophys. 66,752-71.

Macrae CN, Hood BH, Milne AB, Rowe AC, Mason MF (2002) Are You Looking at Me? Eye Gaze and Person Perception. Psychological Science 13 (5), 460-464. 
Martin WW, Jones RF. (1982) The accuracy of eye-gaze judgment: a signal detection approach. Br J Soc Psychol. 21 293-9.

Morrone, M.C., Burr, D.C., \& Maffei, L. (1982) Functional implications of crossorientation inhibition of cortical visual cells. I. Neurophysiological evidence. Proc. $R$. Soc. Lond. B 216, 335-354

Senju A, Hasegawa T. (2006) Do the upright eyes have it? Psychon Bull Rev. 13, 223-8. 
Figure legends

Figure 1. Extracts from stimuli used in Experiment 1. The subject of the picture is looking (from top) to her left by $4^{\circ}$, straight ahead, and to her right by $4^{\circ}$. The stimuli used in the experiment showed the entire head.

Figure 2. Results from Experiment 1. Thresholds for eyeball rotation plotted as a function of effective viewing distance, for two stimulus durations (left: $20 \mathrm{msec}$, right: $1000 \mathrm{msec}$ ), and two observers.

Figure 3. Extracts from stimuli used in Experiment 2. The subject of the picture is looking $4^{\circ}$ to his right, with (from top) raised, neutral, and lowered eyebrows. The stimuli used in the experiment showed the entire head.

Figure 4. Results from Experiment 2. Effective viewing distance producing 84\% correct gaze direction responses, as a function of stimulus duration, for three different positions of the eyebrows, and for two observers. See text for interpretation of the open symbols. The error bars indicate 95\% confidence limits.

Figure 5. Results of a simulation of V1 vertically-oriented cell responses to the nasal and temporal sclera, as a function of viewing distance, for raised, neutral, and lowered eyebrows. The units of response are arbitrary, but the scale is consistent between graphs. The eyeball was directed straight ahead. As can be seen the distance at which the response to the temporal sclera vanishes depends on eyebrow state. 
Figure1: Extracts from stimuli used in Experiment 1
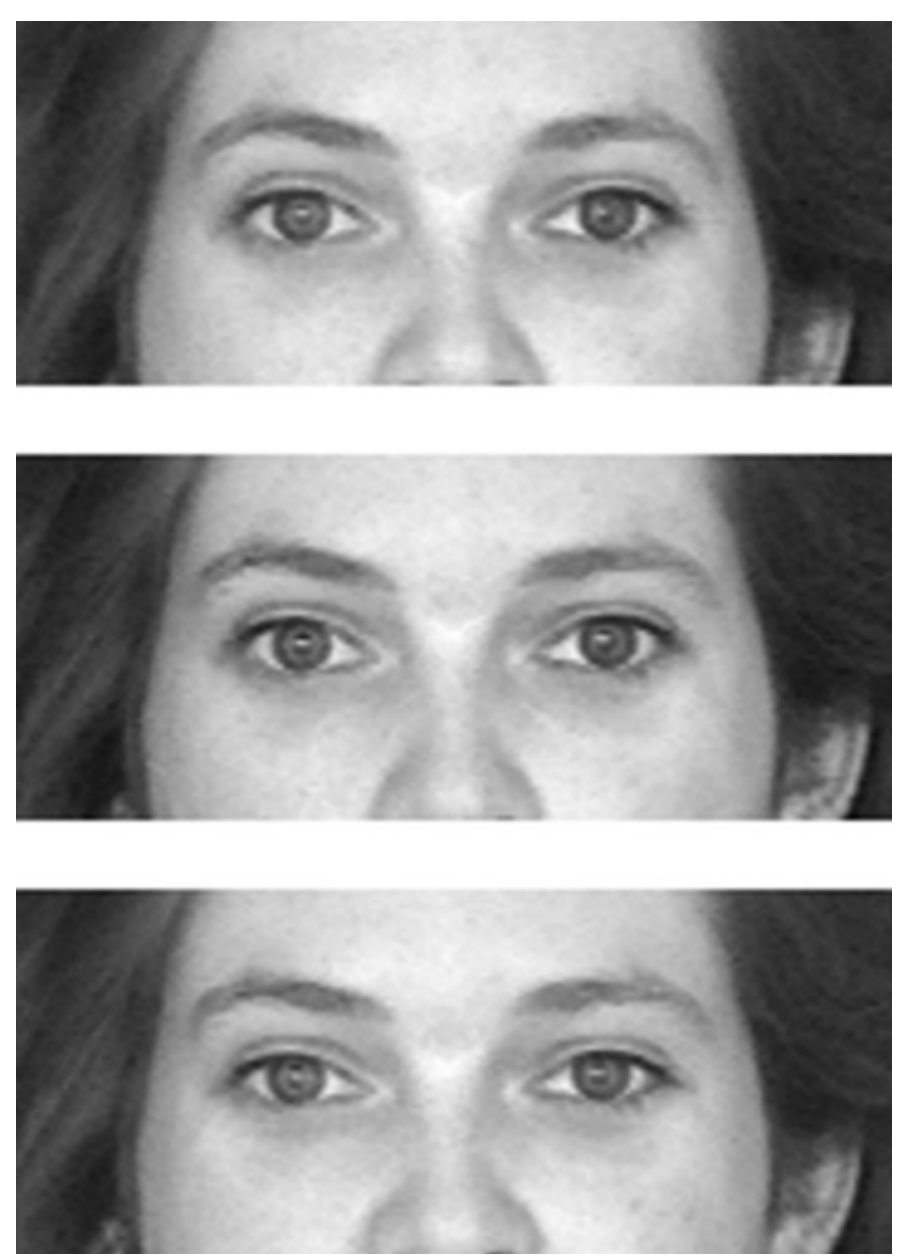
Figure 2. Results from Experiment 1.
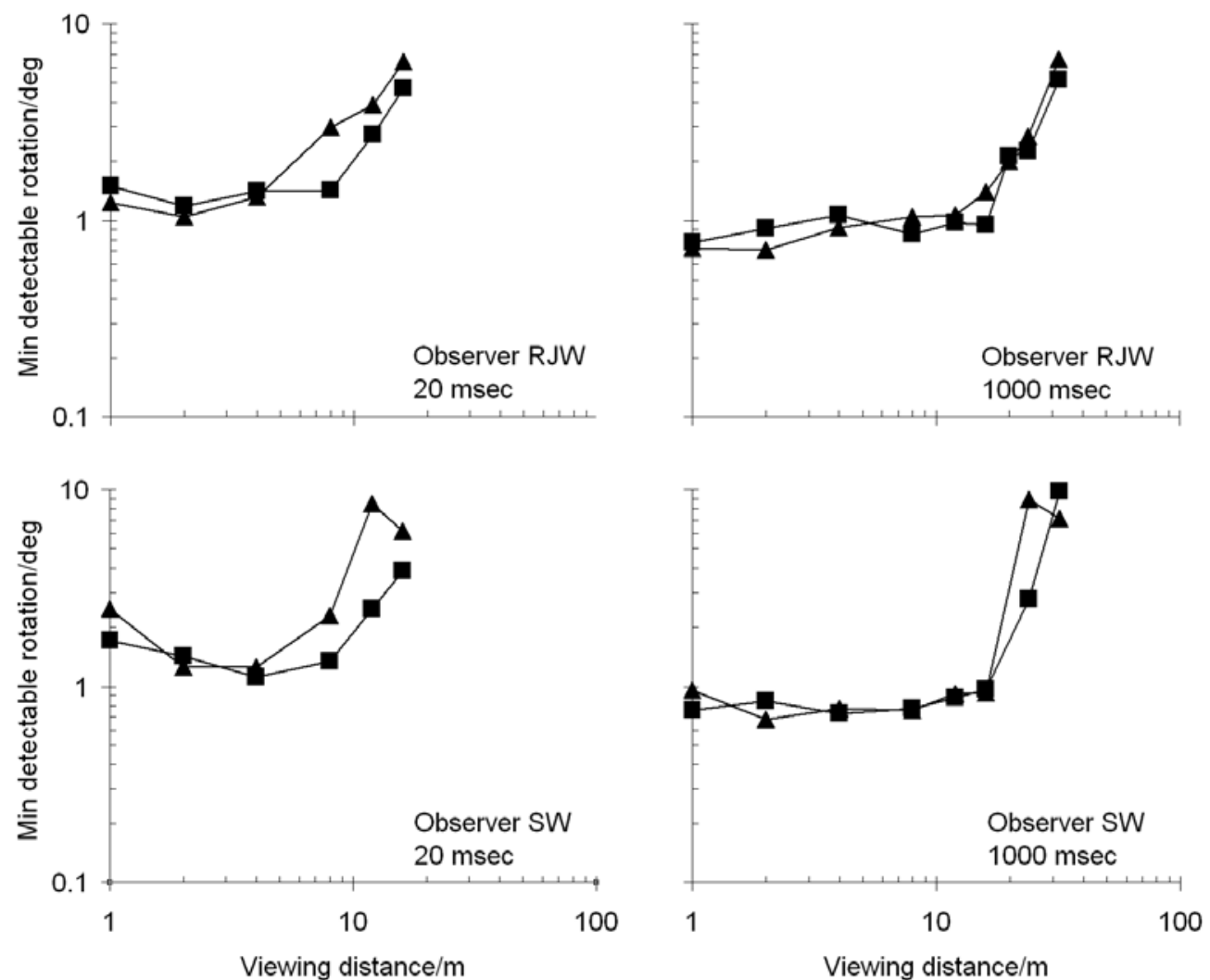

$\rightarrow$ Neutral eyebrows

$\neg$ Low eyebrows 
Figure 3. Extracts from stimuli used in Experiment 2.
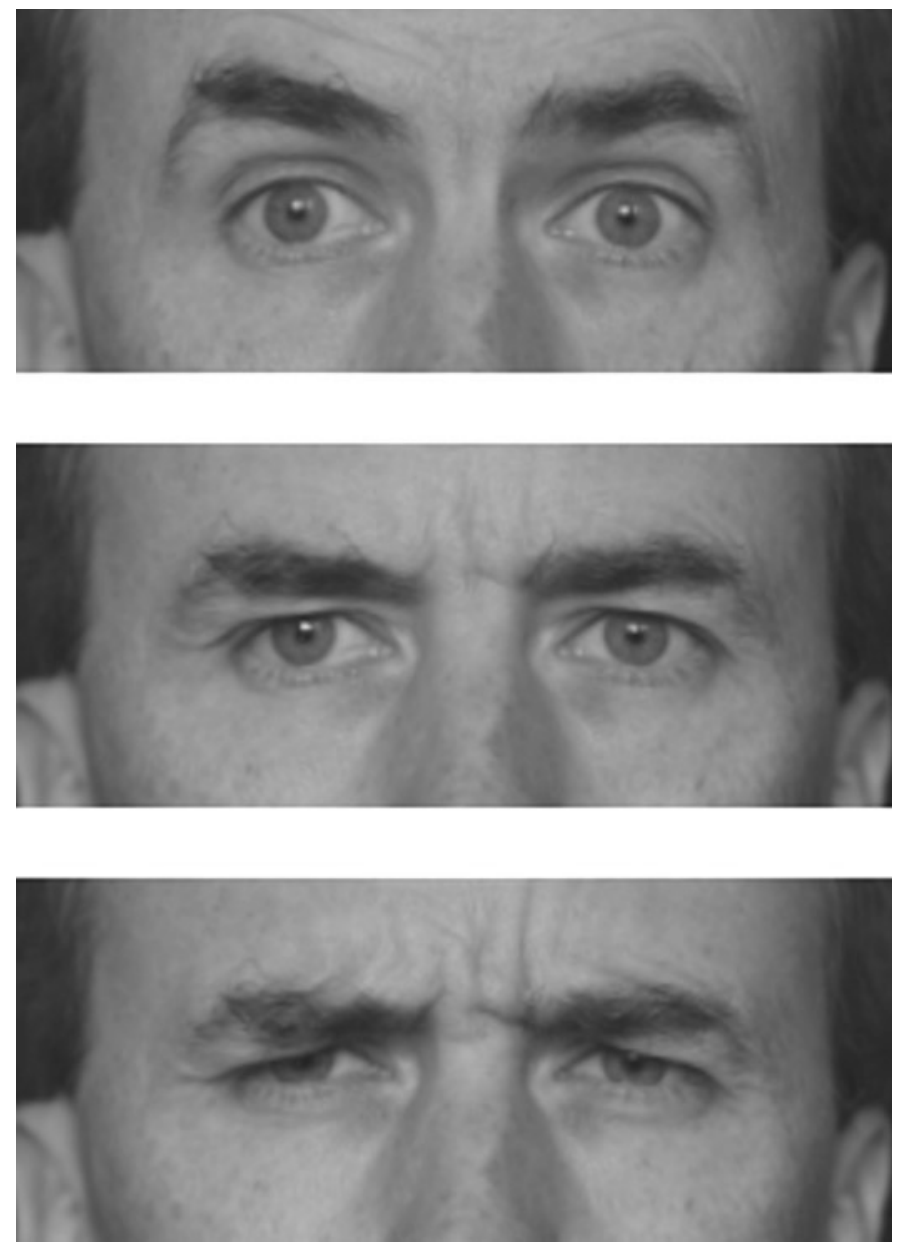
Figure 4. Results from Experiment 2.
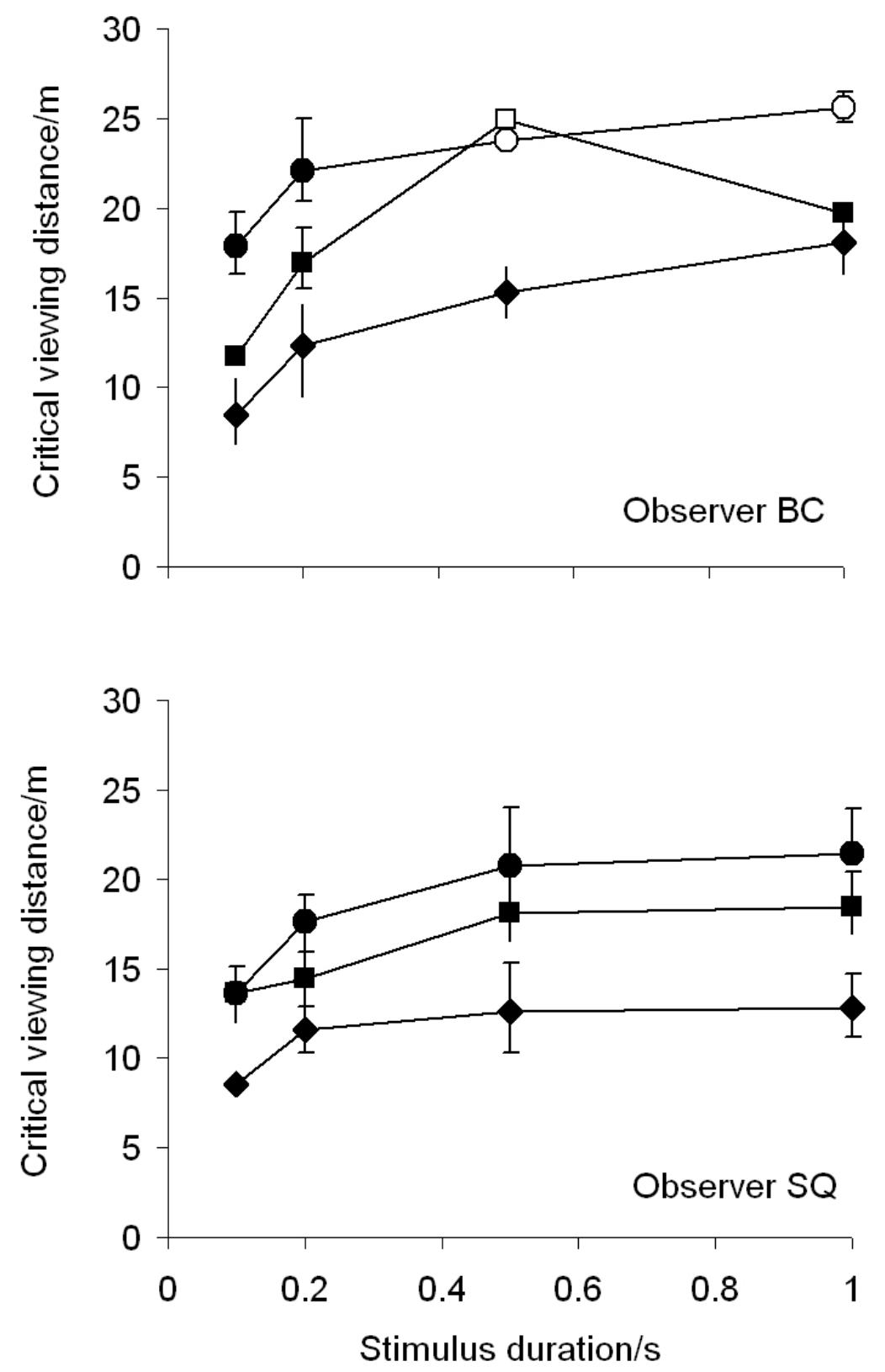

- - High eyebrows

- Neutral eyebrows

Low eyebrows 
Figure 5. Results of a simulation of V1 vertically-oriented cell responses to the nasal and temporal sclera, as a function of viewing distance, for raised, neutral, and lowered eyebrows.
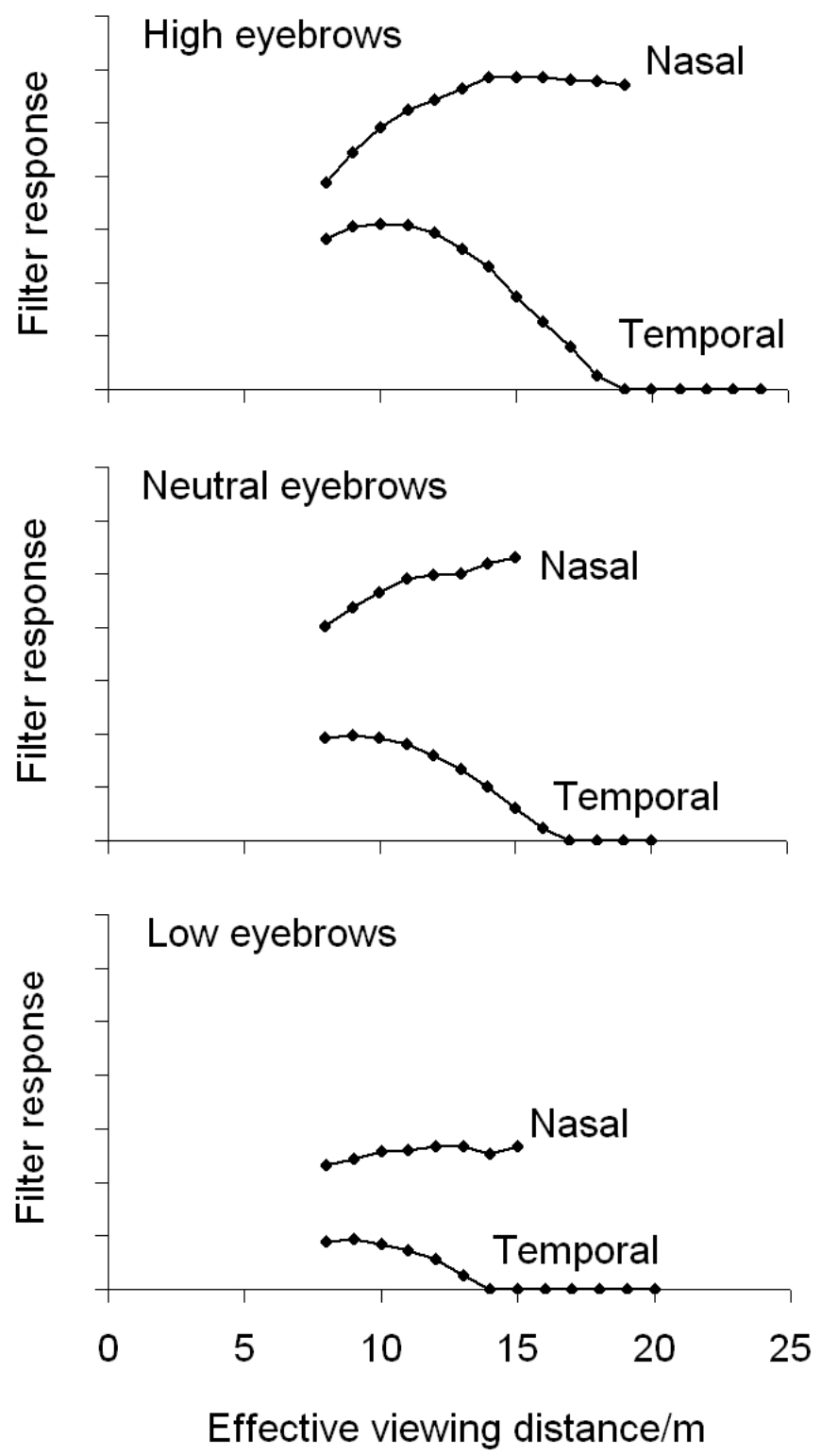